\title{
Dark haloes in Karachentsev's sample of binary galaxies
}

\author{
P. Teerikorpi ${ }^{\star}$ \\ Tuorla Observatory, University of Turku, 21500 Piikkiö, Finland \\ Received 1 February 2001 / Accepted 9 March 2001

\begin{abstract}
Karachentsev's sample of 585 compact binary galaxies is inspected in order to see a signature of extended massive dark haloes around the component galaxies. Our approach utilizes the good velocity-separation resolution provided by this sample containing many close pairs, in order to see directly the expected trends in the $M / L$ vs. $X_{\text {proj }}$ plane. A developed version of Turner's normalization, attempting to take into acount different sizes of binary orbits (increasing with luminosity), makes it possible to see "directly" a meaningful lower limit of $M / L$ from the emerging short-projection envelope in the $M / L$ vs. $X_{\text {norm }}$ diagram. This part of the diagram is little influenced by the incompleteness at large values of separation $X_{\text {proj }}$, which usually leads to too small values of mass-to-luminosity ratios. We conclude that these binaries give relatively direct evidence for the existence of massive dark haloes, with $M / L$ (conservatively) larger than about 25 (for $H_{0}=75 \mathrm{~km} \mathrm{~s}^{-1} / \mathrm{Mpc}$ ), consistent with the simulation studies on Karachentsev's triplets by Chernin \& Mikkola (1991) and on the binaries by Wiren et al. (1996), and other more recent studies of the triplets (Karachentsev 2000).
\end{abstract}

Key words. galaxies: general - dark matter

\section{Introduction}

Massive dark haloes, extending beyond the visible limits of galaxies, have been a part of our extragalactic thinking since the original suggestions by Einasto et al. (1974) and Ostriker et al. (1974) were confirmed by measurements of rotation curves for dozens of spiral galaxies by Rubin et al. (1976). It is generally thought that the fraction of dark matter, measured in terms of the mass-to-luminosity ratio $M / L$ (in units of $M_{\odot} / L_{\odot}$ ), increases from inner parts of galaxies to galaxy groups. So in small triple galaxy systems one has inferred $M / L$ around 30-60 (Chernin \& Mikkola 1991; Karachentsev 2000). For individual spiral galaxies Bahcall et al. (1995) approximate $M / L$ as a function of the radius $R$ as $45(R / 100 \mathrm{kpc})$.

In this respect, compact binary galaxies have offered an interesting case. The sample collected by Karachentsev, with redshifts largely measured by himself with the $6 \mathrm{~m}$ telescope, constitutes very useful material for studies of the double galaxy mass problem. Karachentsev's sample has been given in his book (in Russian) Karachentsev (1987), while an English summary of his analysis may be read in Karachentsev (1990). The sample consists of 585 pairs, selected using a magnitude limit $\left(m_{1}, m_{2}<15.7\right)$ and an isolation criterium utilizing angular diameters of galaxies, their angular separations, and angular distances

* e-mail: pekkatee@astro.utu.fi from significant neighbouring galaxies. The isolation criterium leads to quite compact pairs.

Karachentsev concluded that his sample unexpectedly does not give evidence for extensive dark matter haloes around the galaxies. Specifically, he derives for different types the following mass-to-luminosity ratios, in round numbers: ${ }^{1}$ E: 10; S0: 11; Sa: 9; Sb: 9; Sc: 5; Sm: 5.

In view of the notable separations of even these compact pairs (many have $r_{\text {proj }}>50 \mathrm{kpc}$ ), the mass-toluminosity ratios around 10 seem to contradict the presence of massive haloes as inferred from individual galaxies and from triples and groups. Karachentsev (1990) suggests that mergers of double galaxies operate selectively so that the galaxies with large, massive haloes become more quickly consumed, and hence what is left are binaries with smaller haloes.

Then Wiren et al. (1996) compared computer simulations of interacting galaxies in compact groups with the observed distributions of the velocity differences $(F(\Delta V))$ and projected separations $\left(G\left(X_{\text {proj }}\right)\right)$ of Karachentsev's binaries. They concluded that within their merger model the results are consistent with $M / L \approx 50$ for the binaries. Although the reason for the previously-derived small $M / L$ was left open, it was argued that 10 and 50 may define lower and upper limits for the true $M / L$. The simulation study by Byrd \& Valtonen (2001) on tidal

\footnotetext{
${ }^{1} H_{0}=75 \mathrm{kms}^{-1} / \mathrm{Mpc}$ as Karachentsev had; $M / L \propto H_{0}$.
} 
perturbations and Markarian activity in Karachentsev's binaries also supports extended haloes. It is clearly worthwhile to take a new look at Karachentsev's doubles to see if, after all, one can in some more direct manner discern a sign of the behaviour due to massive haloes.

Instead of sophisticated methods which attempt to take into account the selection effects hampering the determination of the average binary masses (e.g. Turner 1976a, 1976b; Schweizer 1987), our aim is to find a simple way of detecting massive haloes, if they exist, in the large Karachentsev sample. We follow the philosophy which has proved useful elsewhere - the method of normalized distance in the search for the value of the Hubble constant. There one avoids uncertain corrections by putting the data in a form where $H_{0}$ is almost "directly" seen in an "unbiased plateau". Something like that is attempted here for the double galaxy mass-to-luminosity ratio.

In the analysis we take advantage of the fact that compact doubles are well represented in Karachentsev's sample, which allows one to see "with a good resolution" the expected trends at small projected separations.

\section{The incompleteness at large separations}

Karachentsev emphasized that the sample is incomplete at large projected separations, and he gives a formula for the incompleteness as a function of $X$ (the projected separation), based on simulations: $1-\exp (-X / 45 \mathrm{kpc})$. We take this formula as a rough first-order indicator of the incompleteness, seen to reach 90 percent at $X=100 \mathrm{kpc}$. This means that only 10 percent of potential doubles around this $\mathrm{X}$-value have entered the sample. We mean here by "potential" such doubles which have small-X counterparts in the data (i.e. otherwise similar but viewed with a larger inclination angle). The incompleteness has a large influence on any conclusions about the dark haloes. In fact, the problem of incompleteness is at least as important as the problem of false pairs. Just excluding false pairs would leave the calculated mass-to-luminosity ratios too small.

The incompleteness is clearly seen in the behaviour of the limiting magnitude as a function of the linear separation $X$. Figure 1 shows that the limit decreases from $16^{m}$ at $X \approx 0$ to less than $15^{m}$ around $X \approx 200 \mathrm{kpc}$. This cannot be directly transformed into incompleteness ratio using the standard $10^{-0.6 \Delta m}$-factor, because one expects that not only the magnitude limit changes, but also the selection function at all $m$ decreases when the separation $X$ increases.

Any pair of points at small $X$, coming from a true double, should have counterparts (with same magnitudes) at larger values of $X$, up to $X_{\max }=$ the size of the orbit, otherwise any such subsample of binaries is bound to provide an unbiased measurement of the mass. In what follows, we do not need detailed knowledge of the incompleteness, but it prompts us to find another approach to the binary mass problem.

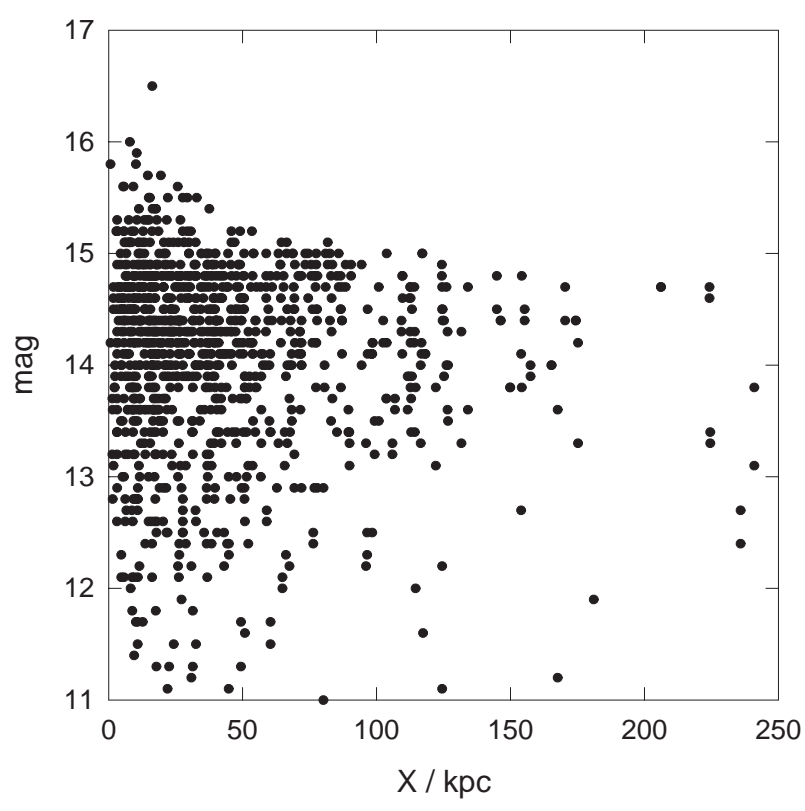

Fig. 1. The behaviour of the limiting magnitude versus projected distance $X$. Note that the limiting magnitude decreases at large separations and that faint, wide pairs have only their narrow projections in the sample

Before turning to the data themselves, we first summarize what would be expected, in terms of observable quantities, from a complete sample of binary galaxies.

\section{Expected trends in the $\Delta \mathbf{V}-\mathbf{R}_{\text {proj }}$ data}

Now we look simultaneusly at the velocity difference $\Delta V$ and the projected separation $X$. These quantities give the raw mass of a pair, $\propto X \Delta V^{2}$. Numerical experiments under different assumptions on the excentricity of the orbit give examples of how the different quantities are distributed for observed doubles with random orientations in space. Figure 2 shows for circular orbits the (normalized) $\Delta V$ vs. $X$ diagram (a) and the raw mass vs. $X$ diagram (b).

Recall that if one uses Kepler's III law without any projection factors, then only the sharp upper corner point gives the correct mass. Everywhere else it is underestimated. Of course, generally an average projection factor $(>1)$ is used in order to give an average true mass for a sample free of selection effects. For circular orbits this factor is $32 / 3 \pi=3.395$. Clearly, for any subclass of identical double galaxies with circular orbits, one expects to see the triangle in the $\Delta V$ vs. $X$ plane filled by points.

In reality, the excentricity may differ from zero. Figure 3 shows the case of $e=0.25$ for identical doubles. If the galaxy masses and orbit sizes span a range of values, the observed $\Delta V$ vs. $X$ diagram is a sum of such different subclasses, and the present author has found it helpful to think of the double galaxy data in terms of several "triangles" with their tips at $\Delta V=0, X=0$.

The selection criteria exclude a fraction of pairs. Only for a complete (or unbiased) subsample of identical 

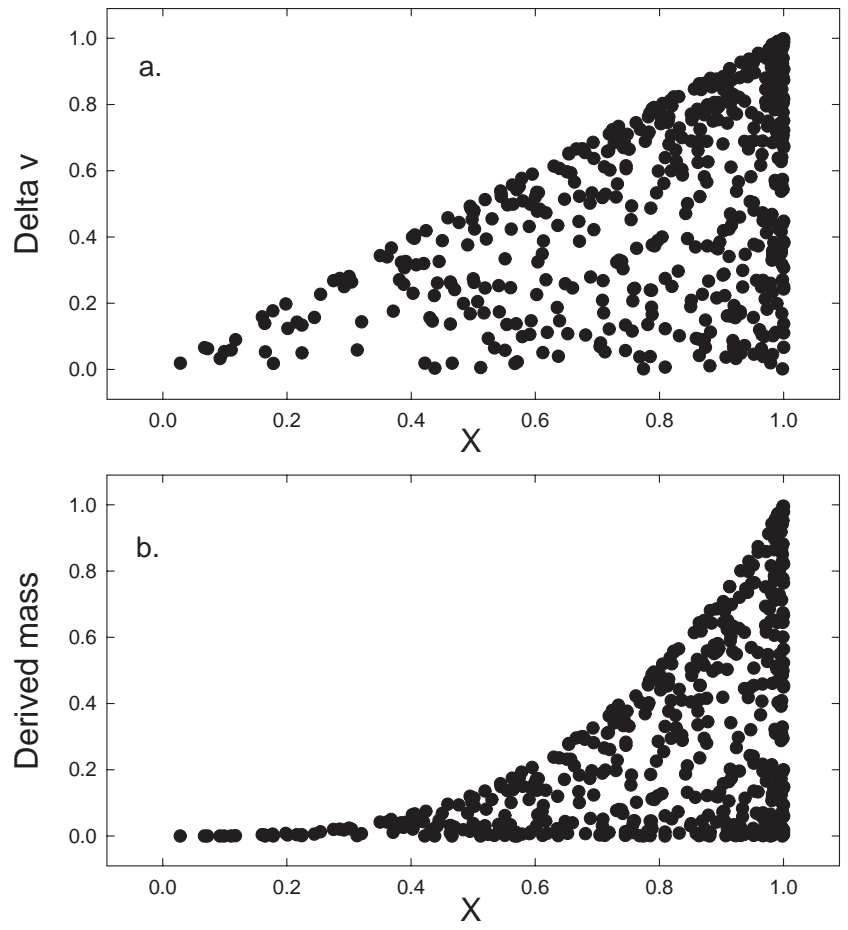

Fig. 2. a) The (normalized) $\Delta V$ vs. $X$ diagram expected for identical binaries with circular orbits. b) The derived raw masses for the above binaries

doubles does one derive a statistically true value of the mass. For those subsamples ("triangles") where large projected distances are lacking, one derives average masses which are too small.

As mentioned above, there are many subclasses contributing to the $\Delta V$ vs. $X$ diagram and we assume that these define an upper envelope in the diagram.

\section{Inspection of the data: Optical pairs}

We first look especially at pairs containing spirals from $\mathrm{Sa}$ to Sc, in order to recognize optical interlopers from the Hubble trend in their velocities and magnitudes. Because the distances are inferred from the Hubble law, we restrict the radial velocity to $>500 \mathrm{~km} \mathrm{~s}^{-1}$.

Figure 4 shows the major part of the $\Delta V$ vs. $X$ diagram. One can make the following observations:

1) At small separations one sees the pattern roughly as expected on the basis of Figs. 2 and 3 (note: there is not yet any normalization here). 2) Furthermore, there is evidence that this pattern continues as a horizontal envelope above $X=50 \mathrm{kpc}$, around $\Delta V=400 \mathrm{~km} \mathrm{~s}^{-1}$. 3) There are some data points clearly scattered above this main distribution, especially below $X=50 \mathrm{kpc}$. These are generally not true binaries. Figure 5 a shows that these pairs have the magnitude difference $m_{2}-m_{1}$ correlated with the $(\log )$ velocity difference $\log \left(v_{2} / v_{1}\right)$ in the sense expected from the Hubble law. The pairs below the gap in the $\mathrm{d} V$ vs. $X$ diagram are randomly distributed (Fig. 5b; however, here the velocity differences are so small that one would not expect to see any similar correlation).
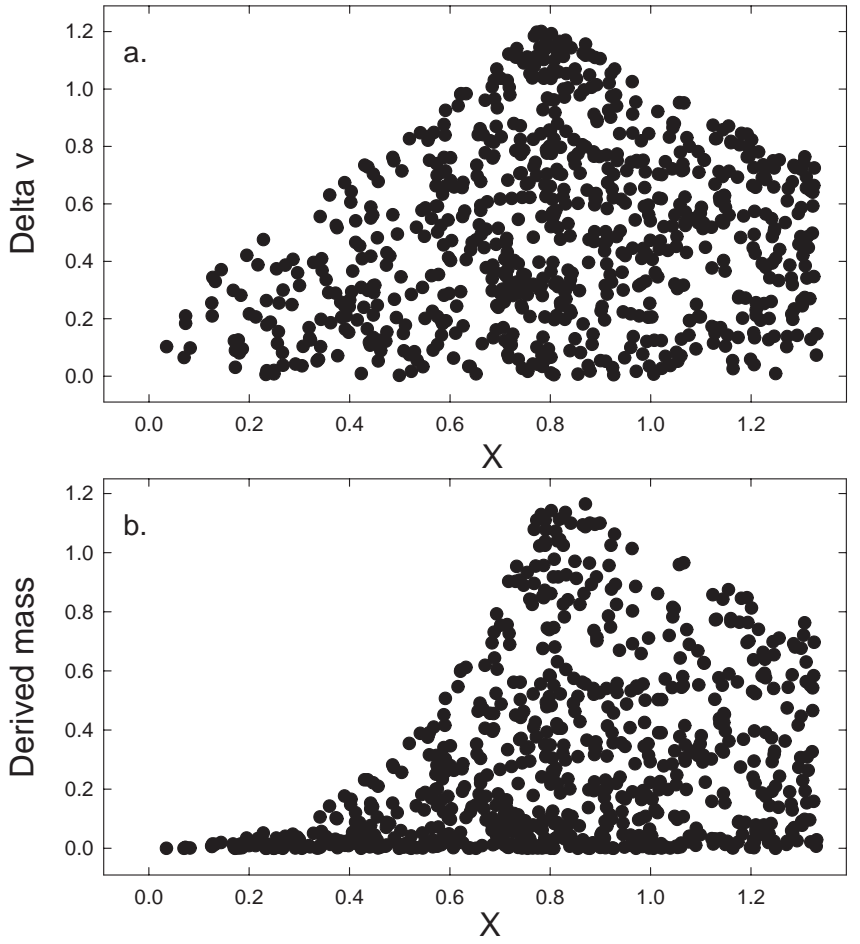

Fig. 3. a) The $\Delta V$ vs. $X$ diagram expected for identical binaries having excentric orbits with $e=0.25$. b) The derived raw masses for the above binaries. Now there is a maximum in the raw mass slightly larger than 1 (= the true mass)

One may ask why there is such a concentration of false pairs at small projected distances, while one might expect such cases especially at increasing distances? The reason for this must be sought from the selection criteria which favour small galaxies at small angular separations, and which is well seen in the behaviour of the limiting magnitude (Fig. 1). The number of (background) galaxies increases when the size decreases and the probability of having an accidental configuration in the sky fulfilling the isolation criterium, is increased. Fortunately, such false pairs have typically large velocity differences which allow them to be (more or less) identified in the $\Delta V$ vs. $X$ diagram.

We add data from the southern sky, by Sadler \& Sharp (1984) and Reduzzi \& Rampazzo (1995) who used about similar selection procedure as Karachentsev. These galaxies ( including also S0-S pairs) have been added in Fig. 4, and they enforce the impression from Karachentsev's data. There also is the study of small satellite galaxies (Zaritsky et al. 1993) which indicated an envelope around $275 \mathrm{~km} \mathrm{~s}^{-1}$ at this $X$-range. The satellites were small (typically $\Delta m>2.5 \mathrm{mag}$ ), hence they were thought to trace the rotation curve of the single central galaxy. In Karachentsev's sample the components are more comparable to each other, hence the rotation curve should reflect masses about twice those of the host \& small satellite case. If one multiplies $275 \mathrm{~km} \mathrm{~s}^{-1}$ by $\sqrt{2}$, the resulting $390 \mathrm{~km} \mathrm{~s}^{-1}$ is close to the value seen in the present sample. We note that Turner (1976b) proposed $425 \mathrm{~km} \mathrm{~s}^{-1}$ 


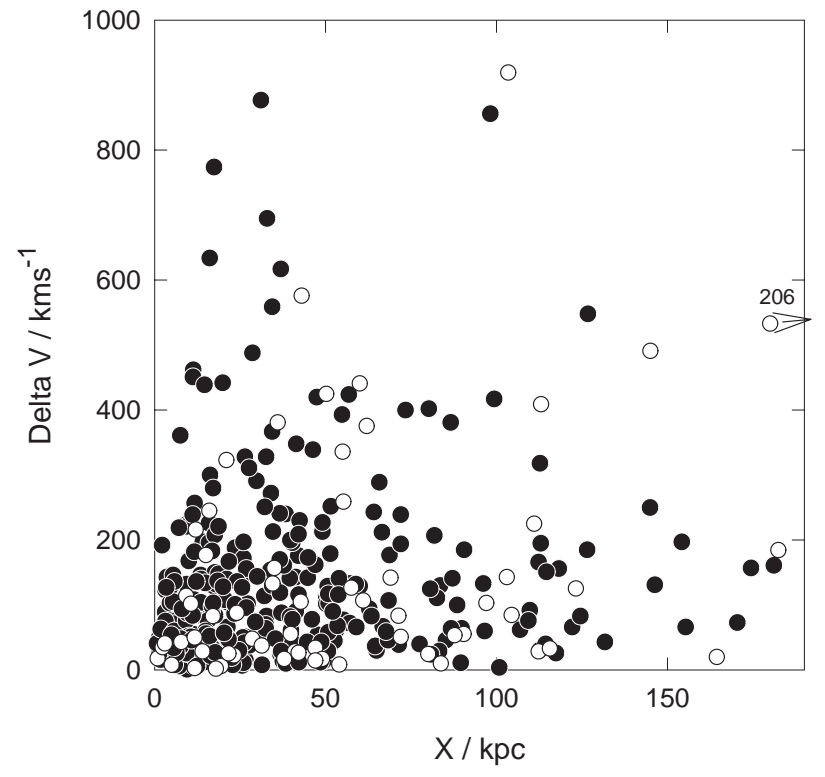

Fig. 4. Velocity difference $\Delta V$ versus projected distance $X$ for spiral pairs with magnitude difference less than 3.0 mag. Open circles: additional pairs from Sadler \& Sharp (1984) and Reduzzi \& Rampazzo (1995). Several pairs with $\Delta V>$ $1000 \mathrm{~km} \mathrm{~s}^{-1}$ and a few pairs with $X>190 \mathrm{kpc}$ (the latter are all below the emerging envelope), are not shown. As discussed in the text, the high velocity-difference galaxies above the gap are probably false pairs

as a dividing line between true and optical pairs in his sample.

\section{The normalized $\Delta V$ vs. $X$ diagram: Check for luminosity dependence}

The next step is to try a normalization of the $\Delta V$ vs. $X$ diagram, following Turner (1976b) who noted that the velocities may be brought to a common level, dividing them by $(\text { luminosity })^{1 / 2}$, because mass $=$ const. $X \Delta V^{2}$. However, this procedure is only valid if 1) the mass-toluminosity ratio does not depend on galaxy type and 2) if the observed luminosity also is proportional to the dark mass component and 3) if the orbit sizes of weak and luminous pairs are similar.

Hence we use in the normalized $\Delta V_{\mathrm{n}}$ vs. $X$ diagram the simple-minded normalization from the total luminosity calculated as

$L_{\text {tot }}=10^{0.4(M 1+20)}+10^{0.4(M 2+20)}$.

This conveniently gives $L_{\text {tot }}$ in the units of the luminosity of a galaxy with absolute magnitude $=-20{ }^{2}$

Figure 6 shows the thus-normalized $\Delta V$ vs. $X$ diagram, excluding the probable false pairs above the envelope. Dots indicate luminous $\left(L_{\text {tot }}>2.0\right)$, open circles

\footnotetext{
${ }^{2}$ If the total, asymptotic $M / L$-ratio depends on type, then a more relevant normalization would need $L=$ $\beta_{1}(T 1) 10^{0.4(M 1+20)}+\beta_{2}(T 2) 10^{0.4(M 2+20)}$, where $\beta$ gives the dependence of the mass-to-luminosity ratio on type.
}
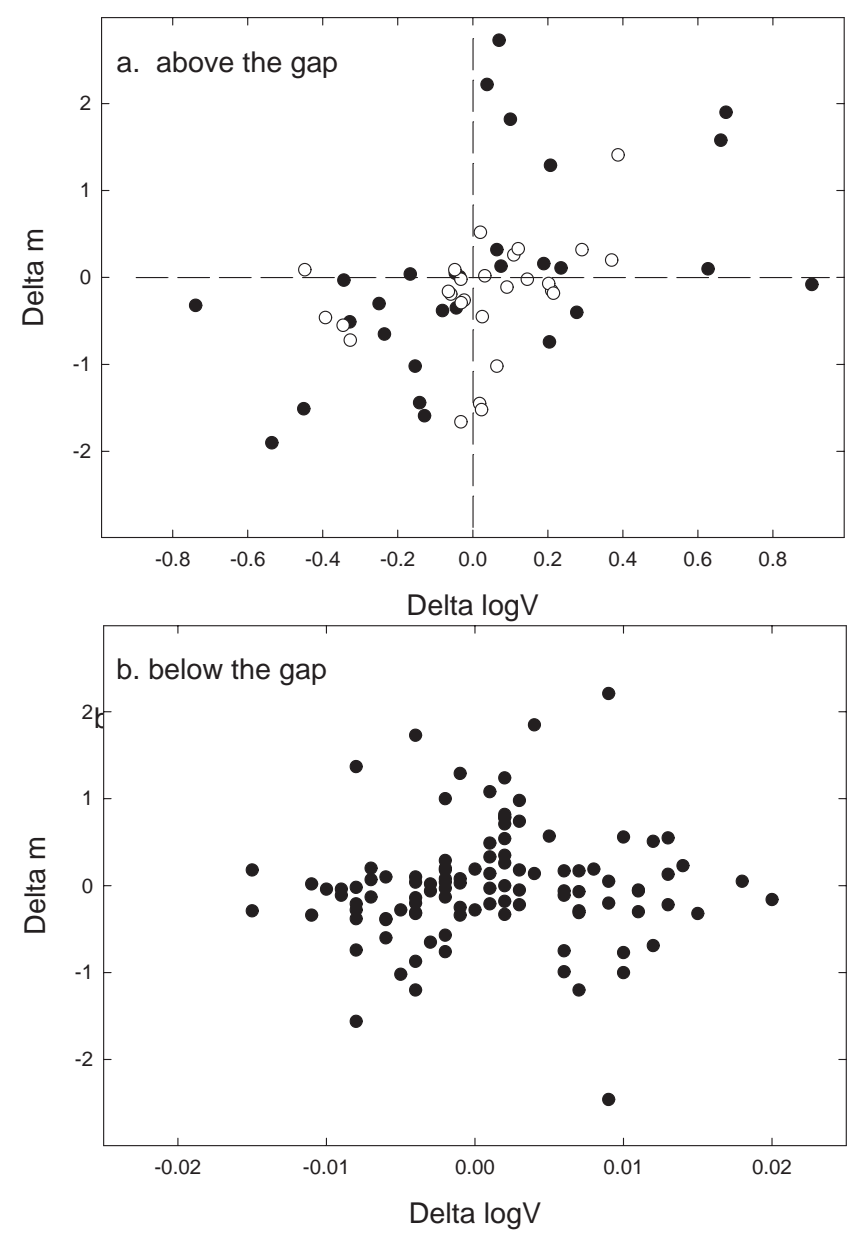

Fig. 5. Magnitude difference vs. (log) velocity difference for Karachentsev pairs above a) and below b) the gap in Fig. 4. In a) the open circles are very distant binaries, $V>7500 \mathrm{~km} \mathrm{~s}^{-1}$. Note the clear Hubble trend in a)

fainter pairs. Note that there are many faint ones scattered above $\Delta V_{\mathrm{n}}=200 \mathrm{~km} \mathrm{~s}^{-1}$ and above the envelope defined by the more luminous ones.

What are those scattered pairs? Apparently the normalization is inadequate: these pairs have small projected sizes which could mean that they are drawn from a population with small intrinsic sizes.

\section{The normalized $M / L$ vs. $X$ diagram}

Then, in order to obtain a correct normalization in the (small $X$ ) growing envelope of the $M$ vs. $X$ diagram, one must add to the procedure a size term. Here we at once go to $M / L$, which automatically incorporates Turner's normalization, but still requires size normalization.

We illustrate the effect by considering the relation luminosity (mass) $\propto \operatorname{size}^{\alpha}$, where $\alpha$ is first taken to be 2 , and normalizing $X$ (norm) $=L^{1 / 2} X$. In the thus-normalized $M / L$ vs. $X$ diagram in Fig. 7 both luminous and faint binaries now define a rather sharp common envelope rising up to $M / L \approx 35$, after which there are a few still higher values around the continuation of this envelope curve. 


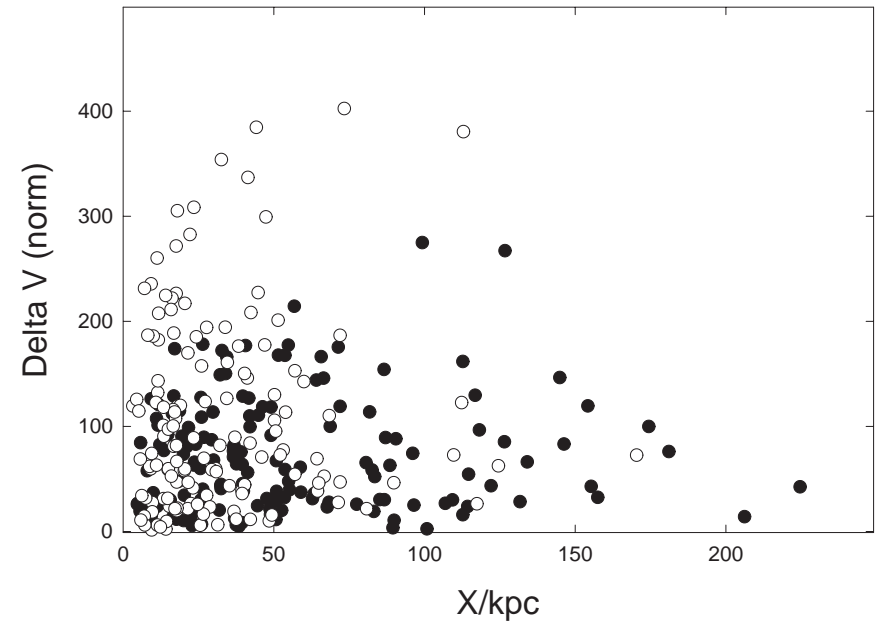

Fig. 6. The normalized $\Delta V_{\mathrm{n}}$ vs. $X$ diagram for luminous (dots) and fainter (open circles) pairs

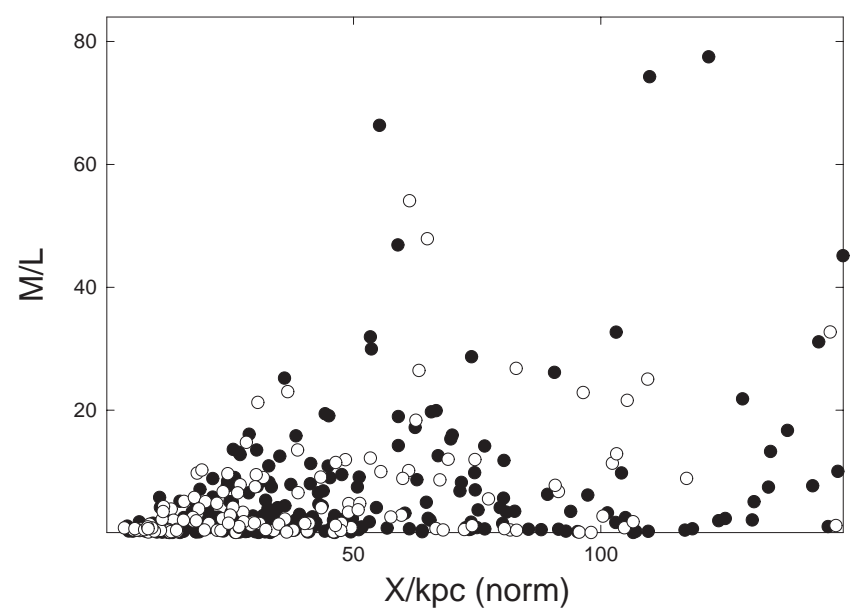

Fig. 7. $M / L$ vs. normalized $X$. Dots are luminous binaries $(L>2)$, open circles are fainter $(L<2)$. All galaxy types are included

Note that false pairs due to projections inside groups will not follow the normalization. If a good envelope appears, this is a sign that such false pairs do not distort it. Inspection of the $M / L$ vs. $X_{\text {norm }}$ diagram shows that the common envelope is defined, not only by faint and luminous binaries, but also by different Hubble types.

Figure 8 gives the leftmost part of the diagram up to $X_{\text {norm }}=50 \mathrm{kpc}$, showing how remarkably well the adopted normalization works down to small projections. In Fig. 9 we added open triangles representing the simulated expected distribution for the case $e=0.25$. The simulated points have been adjusted to $M / L=50$ and $R_{\text {norm }}=60 \mathrm{kpc}$. One can see that the observed envelope is close to that expected from randomly oriented binaries having orbits with about such an eccentricity.

However, we prefer to conclude more conservatively that the emerging envelope at short $X$ gives evidence for $M / L>25$, and thus is consistent with the results obtained for Karachentsev's triple galaxies and small groups.

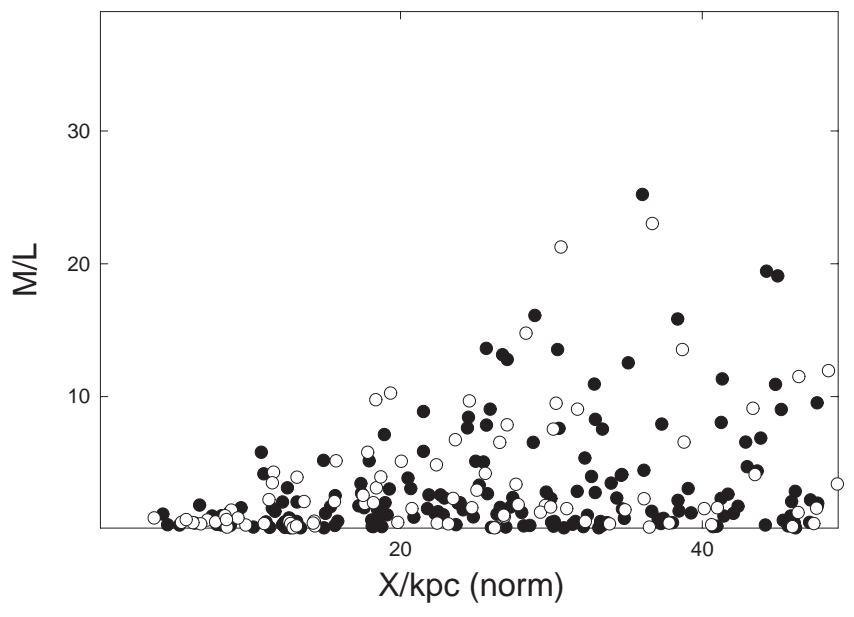

Fig. 8. The leftmost part of the $M / L$ vs. normalized $X$ diagram. Dots are luminous binaries $(L>2)$, open circles are fainter $(L<2)$. All galaxy types are included

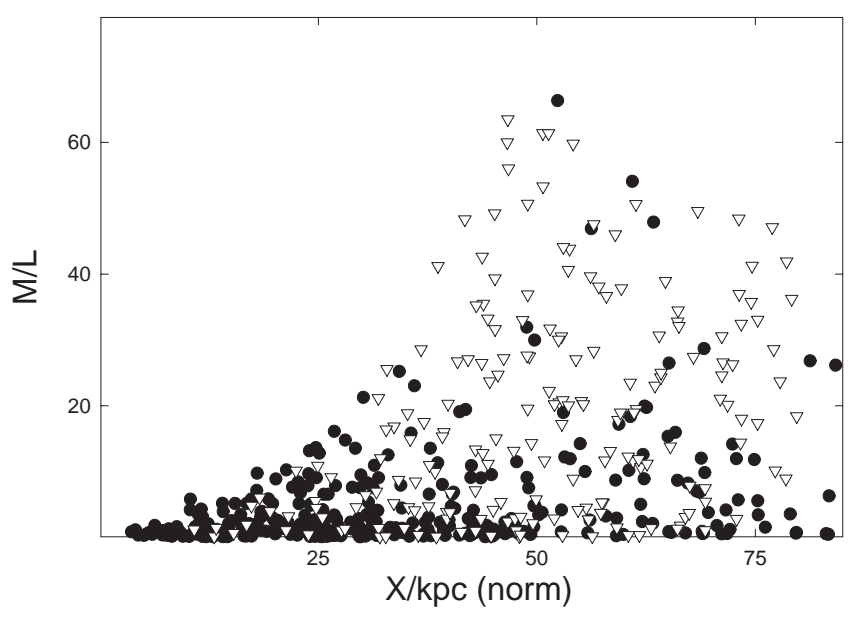

Fig. 9. The short- $X_{\text {norm }}$ part of the $M / L$ vs. $X_{\text {norm }}$ diagram. Open triangles are simulated binaries having excentricity $e=$ 0.25 and $M / L=50$. Dots are real binaries

\section{Discussion and conclusions}

After a succesful normalization, the extension of the left envelope of the $M / L$ vs. $X_{\text {norm }}$ diagram can be considered as giving a lower limit for the $M / L$, not affected by the incompleteness at large separations. Indeed, the appearance of the envelope is the best way to see that

* binary galaxies rotate around each other, and

* have massive haloes,

leading to $M / L$ ratios appproaching those of triplets. The incompleteness at large projected $X$ does not influence the run of the short- $X_{\text {norm }}$ envelope, though it makes the envelope increasingly poorly defined at large $X_{\text {norm }}$. It is this incompleteness which leads to a biased value of $M / L$, if one calculates the average mass-to-luminosity ratio, using a standard projection correction factor.

Now Fig. 8 shows that $M / L$ reaches at least 20 and Fig. 9 suggests that it is possibly as high as 50. One should 
note that the first limit already is consistent with the $\mathrm{S}$ galaxy value $\approx 20$ required by the simple disk + bulge + dark halo model, which explains the type dependence of the $B$ mag TF relation (Theureau et al. 1997), up to the radius where the rotation is effectively measured.

In fact, these $M / L$ values from the extension of the envelope are slightly biased upwards, because they do not include the correction due to velocity measurement errors. As the envelope is defined by galaxies typically having $\Delta V>200 \mathrm{~km} \mathrm{~s}^{-1}$ and the typical error in the velocity difference is $\sigma_{\Delta V} \approx 60 \mathrm{~km} \mathrm{~s}^{-1}$ (Karachentsev 1987), this correction is less than 10 percent.

But what about the binaries scattered to larger values of $X_{\text {norm }}$ than expected on the basis of excentrities 0.25 or even higher? Here one may point out that the normalization was based on the assumption that $M=k R^{\alpha}$. If actually there is simply a general condition that $M \leq k R^{\alpha}$, then the left envelope still appears when this normalization is used. For the binaries with " $<$ " the normalization will be insufficient and they are left at too large $X_{\text {norm }}$.

We can only speculate on the origin of such a relation or restriction. It is in the sense that for a binary with a given separation, the sum of the halo masses (and their sizes) cannot exceed a limit, otherwise perhaps the binary will quickly merge under the influence of dynamical friction. Ostriker \& Turner (1979) found evidence for such a process leading to a size - luminosity dependence, also discernable in galaxy triples (Karachentsev et al. 1999). Then the sharp envelope testifies that Karachentsev's sample is really made of truly compact binaries, close to the allowed limit $R \geq(M / k)^{1 / \alpha}$.

Finally, we note that binaries with one or two elliptical galaxies do not show higher values of $M / L$, contrary to what one might expect from measurements of individual elliptical galaxies (Bahcall et al. 1995). It has been suggested that ellipticals in such compact binaries are end results of merged spiral galaxies in a previous multiple galaxy system (e.g. Wiren et al. 1996). Indeed, in that case the new E galaxies are expected to have a $M / L$ similar to that of their parent galaxies.
Acknowledgements. I am grateful to the referee Igor Karachentsev for several valuable comments. I thank Arthur Chernin, Boris Komberg, and Mauri Valtonen for useful remarks and information. I thank Lena Teerikorpi for good help putting Karachentsev's sample into the computer. This study has been supported by the Academy of Finland (project "Cosmology from the local to the deep galaxy universe").

\section{References}

Bahcall, N. A., Lubin, L. M., \& Dorman, V. 1995, ApJ, 447, $\mathrm{L} 81$

Byrd, G., \& Valtonen, M. 2001, AJ, in press

Chernin, A. D., \& Mikkola, S. 1991, MNRAS, 253, 153

Einasto, J., Kaasik, A., \& Saar, E. 1974, Nature, 250, 309

Karachentsev, I. D. 1987, Binary galaxies, in Russian (Nauka, Moscow)

Karachentsev, I. D. 1990, in Paired and Interacting Galaxies, IAU Colloquium No. 124, p. 3, ed. J. W. Sulentic, W. C. Keel, \& C. M. Telesco, NASA Conference Publication 3098 Karachentsev, I. D. 2000, in Proceedings, IAU Colloquium 174 "Small galaxy groups", ed. M. J. Valtonen, \& Ch. Flynn, ASP Conf. Ser., 209, 1

Karachentsev, I. D., Byrd, G. G., Chernin, A. D., \& Valtonen, M. J. 1999, Fund. Cosm. Phys., 20, 383

Ostriker, J. P., Peebles, P. J. E., \& Yahil, A. 1974, ApJ, 193, L1

Ostriker, J. P., \& Turner, E. L. 1979, ApJ, 234, 785

Reduzzi, L., \& Rampazzo, R. 1995, Astrophys. Lett. Com., 30,1

Rubin, V. C., Ford, W. K., Jr., \& Thonnard, N. 1976, AJ, 81, 687

Sadler, E. M., \& Sharp, N. A. 1984, AA, 133, 216

Schweizer, L. Y. 1987, ApJS, 64, 427

Theureau, G., Hanski, M., Teerikorpi, P., et al. 1997, AA, 319, 435

Turner, E. L. 1976a, ApJ, 208, 20

Turner, E. L. 1976b, ApJ, 208, 304

Wiren, S., Zheng, J.-Q., Valtonen, M. J., \& Chernin, A. 1996, AJ, 111, 160

Zaritsky, D., Smith, R., Frenk, C., \& White, S. D. M. 1993, ApJ, 405, 464 